\title{
Camera flash heating of a three-layer solid composite: an approximate solution
}

\begin{abstract}
Camera flash heating and the subsequent thermal wave propagation in a solid composite material is studied using the Laplace transform technique. Full-field rear surface temperature for a single-layer, two-layer and three-layer solid composites are obtained directly from the Laplace transform conversion tables as opposed to the tedious inversion process by integral transform method. This is achieved by first expressing the hyperbolic-transcendental equation in terms of negative exponentials of square root of s/Ŭ and expanded same in a series by the binomial theorem. Electrophoretic deposition (EPD) and dip coating processes were used to prepare three-layer solid composites consisting $\mathrm{ZnO} / \mathrm{Cu} / \mathrm{ZnO}$ and starch/Al/starch respectively. About $0.5 \mathrm{ml}$ of deionized water enclosed within an air-tight aluminium container serves as the third three layer sample (AL/water/AL). Thermal diffusivity experiments were carried out on all the three samples prepared. Using Scaled LevenbergMarquardt algorithm, the approximate temperature curve for the three-layer solid composite is fitted with the corresponding experimental result. The agreement between the theoretical curve and the experimental data as well as that between the obtained thermal diffusivity values for the $\mathrm{ZnO}$, aluminium and deionized water in this work and similar ones found in literature is found to be very good.
\end{abstract}

Keyword: Camera-flash; Laplace inversion theorem; Laplace transforms; Subsidiary equations 